\title{
Design of instrument for measuring the electrical conductivity of ice cores
}

\author{
Qihong Wang ${ }^{1}$, Jingbiao Liu $^{1, \text { a }}$, Chunlei $\mathrm{An}^{2}$, Jianguang Shi ${ }^{1}$
}

${ }^{1}$ College of Electronic \& Information, Hangzhou Dianzi University, Hangzhou, Zhejiang Province, 310018, China

${ }^{2}$ Polar Research Institute of China, Shanghai, 200136, China

aemail: ab@hdu.edu.cn

Keywords: ice core; electrical conductivity measurement; grating scale; servo motor; STM32F103; data collection

\begin{abstract}
For the application of electrical conductivity measurement of ice core in the analysis of ice core processing, foreign hand-held electrical conductivity measurement unit is developed and updated by using servo motor to automatically control electrical conductivity measurement. while the corresponding human-computer interaction platform is designed to display and save the measured data and control the speed and direction of the motor. The system takes STM32F103 processor as the core and combines with ADS1240, grating scale, servo motor, high-voltage module and temperature sensor, realized the functions of data collection, data transmission and data storage. The test shows that the system can accomplish the electrical conductivity measurement of ice core stably. And compared with abroad, it has a higher precision and wider measurement range, which meet the requirements of the design.
\end{abstract}

\section{Introduction}

Polar ice cores has abundant research value. A variety of equipment and technology are used to study ice cores. Solid electrical conductivity measurement (ECM) of ice cores plays an important role in researching into ice core volcano activity, the classification of chronostratigraphy, biomass burning and analysis of chemical compositions of ice core.

The Icefield Instruments Inc. in Canada has developed a hand-held ECM unit in 2009. Compared with foreign hand-held devices, ECM presented in this paper is greatly improved in measuring range and measuring precision. And the use of low temperature resistant servo motor to drive and control measuring electrode excluded the instability of manual measurement and saved manpower. The structure of electrode is designed with a spring can be adapted to the ice surface automatically. If the ice surface is uneven or cracked, the electrode can be automatically adjusted and guaranteed to be in real time contact with the ice surface. Human-computer interaction platform can real-time display the voltage, current and resistance of measuring position of ice cores and draw the curves of the relative displacement of voltage, current and resistance. Heating device was designed to ensure that the equipment can operate normally in a low temperature environment of 40 degrees below zero.

\section{Overview of the System}

1. Detection principle

The most acidic component of the Antarctic ice sheet is $\mathrm{HNO}_{3}$, followed by $\mathrm{H}_{2} \mathrm{SO}_{4}$. The conductive properties of ice core with relation to the concentration of $\mathrm{H}^{+}$in ice cores. The 
correlation between the current $\mathrm{I}$ and $\mathrm{H}^{+}$concentration is exponential. Formula is $\mathrm{I}=\mathrm{A}+\mathrm{B}^{*}\left(\mathrm{H}^{+}\right)^{\mathrm{C}}[2]$ [3] [4]. The value of A, B, C is related to temperature, year, volcanic activity and so on.

2. Design scheme

This equipment works in low temperature environment. Due to some chips can not work in cold temperatures (generally lower than minus 15 degrees Celsius). Therefore, the whole control system is placed in a metal cabin equipped with a heating device. Outside of the metal cabin is equipped with a digital display temperature control device (DS18B20 temperature sensor is used to measure the temperature inside the cabin). The current cabin temperature value can be displayed in real time. Set the temperature to twenty degrees centigrade before heating. And after heating to the set temperature, the motor and system work button can be turned on.

Ice code which need to be measured is fixed to the workbench. Two parallel and $1.5 \mathrm{~cm}$ apart (can be adjusted to other distance) copper electrodes are adjusted to optimal contact with ice surface. Spring is mounted on the electrode. The electrode can adapt to the uneven surface of the ice core. When the system start button is turned on, $1200 \mathrm{~V}$ voltage is between electrodes. The voltage across the ice code is collected by AD chip. Then the data is calculated by main chip and sent to the host computer through the serial port. When the motor switch is open, left and right sport buttons on the workbench can be operated to control the motor forward and reverse to make the electrodes at a certain speed (initial velocity $10 \mathrm{~mm} / \mathrm{s}$ ) moving on the ice surface [4] [5] . Using grating ruler to measure the position of the ice core (initial position is 0 ). PC software synchronous records the current, voltage and resistance. Test results in two dimensional profile is dynamic real-time display in computer software. The ordinate is current, voltage and resistance. The abscissa is the corresponding length of ice core. To reduce random error, the results is repeated measurements of ice core section. Figure 1 shows the model of the whole scheme design.

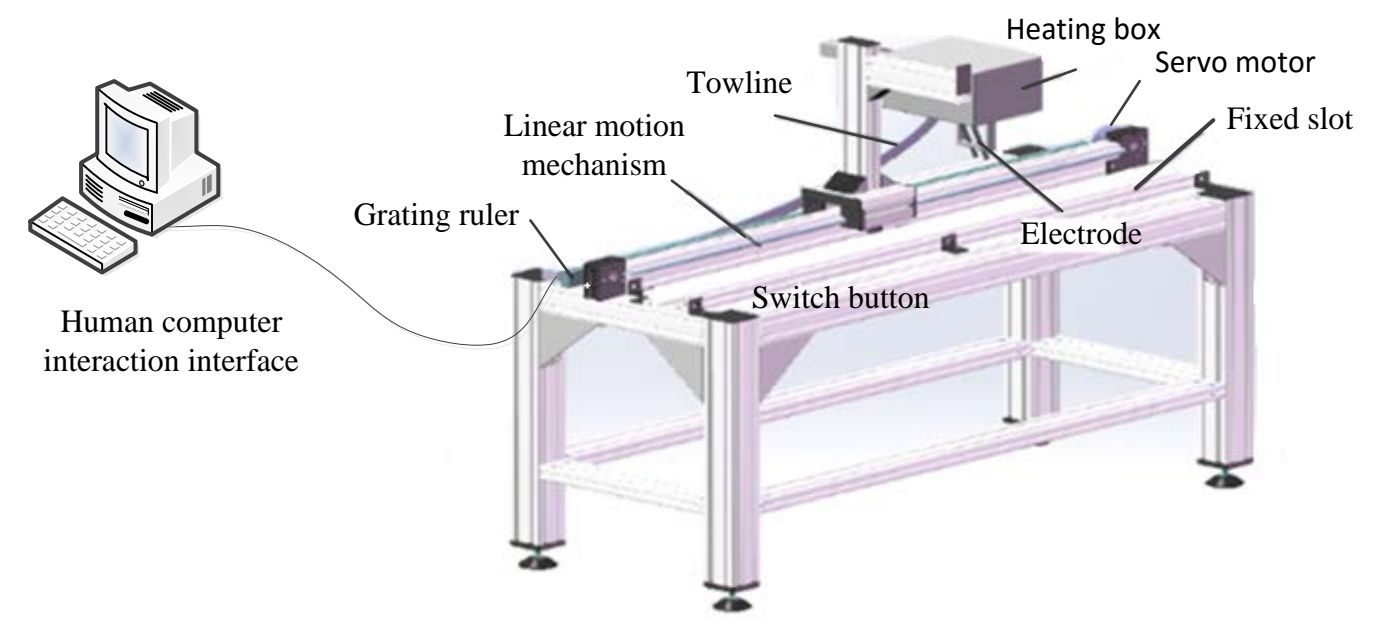

Fig.1. Overall structural design models

\section{Hardware Design}

The overall structure mainly includes six parts: control circuit, high voltage power supply circuit, voltage dividing circuit, analog acquisition and conversion circuit and motor control circuit. Figure 2 shows the hardware design of the whole scheme design. 


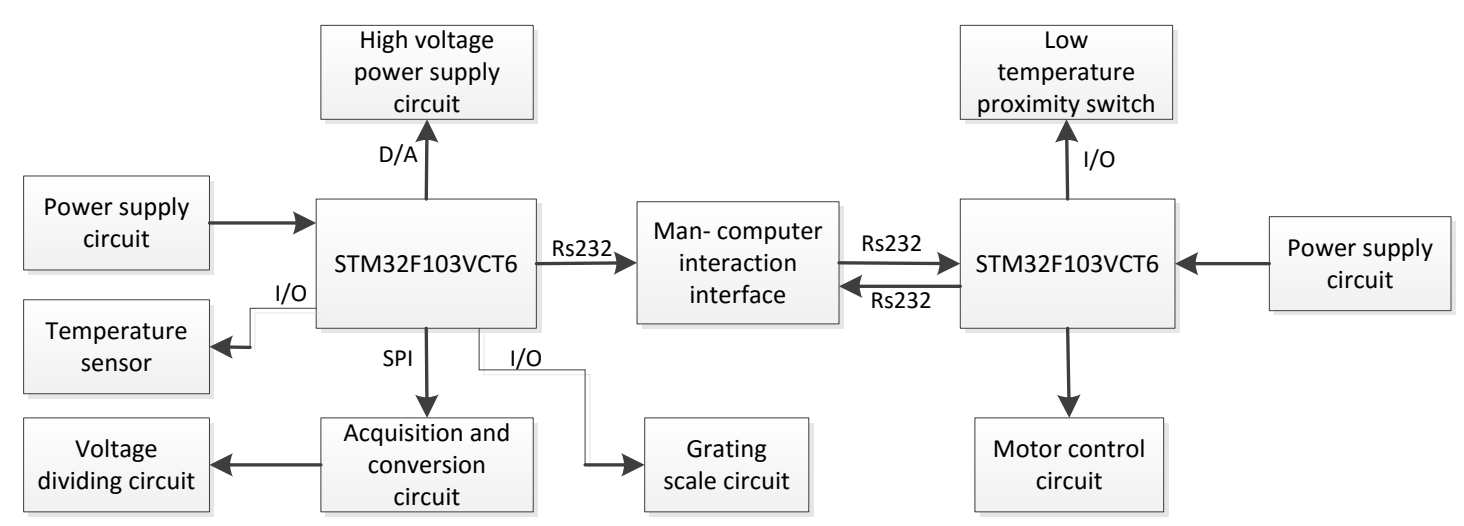

Fig.2. Hardware design diagram

\section{Measurement system circuit design}

The STM32F103VCT6 chip is used as master control chip. It is based on high-performance ARM Cortex-M3 32-bit RISC core operating at a frequency of up to $72 \mathrm{MHz}$ and integrated A/D, D/A, USART, TIMER and other resources.

This system uses the high voltage power supply module to produce the $1200 \mathrm{~V}$ DC voltage. Because if low voltage applied on the ice core surface, the current produced by the voltage dividing is very small because the resistance of ice is very high. 50 megohm resistance is used to protect circuit. To prevent 2 electrodes direct contact and damage circuit.

The value obtained by the voltage dividing circuit is very small. This system uses ADS1240 chip which is produced by TI company to collect voltage. It has up to 24 bit without loss of code characteristics and 21 bit effective resolution. ADS1240 is very appropriate, because the current is microamp range.

The grating scale with high precision is used to measure the displacement position [6]. Input signal of grating scale is differential signal and digital isolator Si8711CC-B is used to isolate the signal.

\section{Motor control design}

In the process of measurement, the stability of electrode movement is critical. Pause will affect the accuracy of the measurement. And the motor must be able to work stably at low temperature.

The motor controller is also take STM32F103VCT6 as the microprocessor. The high speed optocoupler SI8640B is used to isolate the input and power-driven device ULN2803 is used to drive current in circuit design [7].

The motor uses the precision AC servo motor produced by Swiss INFRANOR company. The model is BLS-0408. The motor driver uses the CDHD-0032AAP1 model produced by the SERVOTRONIX company in Israel. Motor speed reducer uses APE60 model produced by German MOTEC company. The reduction ratio is 1:4.

On the workbench, a button box is used to control the motor. And three buttons respectively forward, backward and stop on the button box. At the beginning of the workbench and 1.5 meters from the starting point are both equipped with low temperature proximity switch. The low temperature proximity switch is used for limit and to prevent improper operation damage the equipment.

\section{Software Development}

The software design of the system includes the data acquisition part, the motor part and the host computer software program design. The codes of the data acquisition part and the motor part are written in C language and used RTX operating system. The host computer software program is 
written in C\#.

1 data acquisition design.

1) Main thread

System initialization. Using D/A to control the high voltage module to produce $1200 \mathrm{~V}$ and reading the temperature measured by the temperature sensor.

2) ADS1240 data processing thread

Initialization and configuration for the ADS1240 chip. The collected data is stored in $g_{a b c[0]}$ and $g_{a b c[1]}$ after being processed by filter. Then the voltage and current is calculated. The formula is as follows.

$$
\begin{aligned}
& \operatorname{sigm}_{v}=\frac{g_{a d c[0]}}{0 x 80000000} * \frac{\text { Vref }}{\text { GAIN }} * \frac{\frac{5000}{2}+1}{1000} \\
& \operatorname{sigm}_{i}=\frac{g_{a d c[1]}}{0 x 80000000} *\left(\text { Vref } * \frac{10}{\text { GAIN }}\right)
\end{aligned}
$$

$\operatorname{sigm}_{v}$ is voltage, $\operatorname{sigm}_{i}$ is current, Vref is reference voltage, GAIN is gain.

3) Grating ruler data processing thread

Initialization timer and configuration for encoder mode. Reading and calculating grating ruler data.

4) Data sending thread

The measured data is packaged and sent to the host computer. In accordance with the data frame format “@\#, a, b, c, d, e*\# \r|n”. a is voltage, b is current, c is resistance, $d$ is displacement and e is temperature.

2 Motor program design

1) Main thread

Initialization and configuration. To control the motor by controlling the motor drive when the button is pressed or instructions is transmitted by the host computer.

2) Button processing thread

To judge whether the button is pressed or not.

3) Data receiving thread

The host computer sends the motor control instruction to the microprocessor. the direction of the instruction format is shown in Table 1.

\begin{tabular}{|c|c|}
\hline Command & Action \\
\hline EE 02000600 08 FF & forward \\
\hline EE 0200070009 FF & backward \\
\hline EE 02000900 0B FF & stop \\
\hline
\end{tabular}

Tab.1. Direction instruction

FF, EE is the head and tail of the instruction. The penultimate number is checksum. Speed command is similar to the instruction of direction .

\section{Test Results}

Set the heating temperature to 20 degrees. The equipment is placed in a cold storage of 40 degrees below zero. The ice which length of 1 meters, width of $10 \mathrm{~cm}$ and thickness of $1 \mathrm{~cm}$ is fixed on the V slot. This ice is frozen with pure water. And different concentrations of saline was frozen in different regions of the ice surface.

First, press the heating button to turn on the heating device. After 2 minutes, the temperature in the cabinet is shown to stabilize at 17 degrees to 20 degrees cycle fluctuations. Temperature changes is shown in Figure 3. Then press the master control system button and motor button. The 
forward, backward and stop function of the motor is tested by using the buttons and the host computer. The conductivity curves were plotted in the speed of $5 \mathrm{~mm} / \mathrm{s}, 10 \mathrm{~mm} / \mathrm{s}, 15 \mathrm{~mm} / \mathrm{s}$ and $20 \mathrm{~mm} / \mathrm{s}$ respectively. The curve of current and distance is in Figure 4 . The test results of the host computer at speed of $10 \mathrm{~mm} / \mathrm{s}$ is shown in Figure 5. The overall constructed equipment is shown in Figure 6.

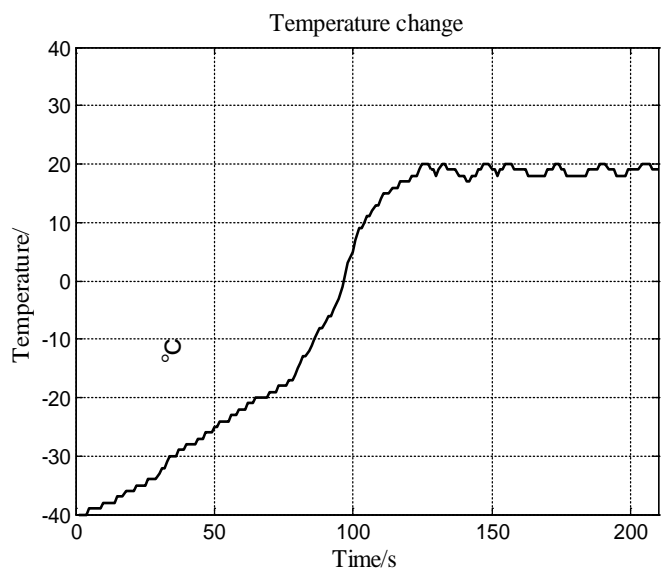

Fig.3. Temperature change curve

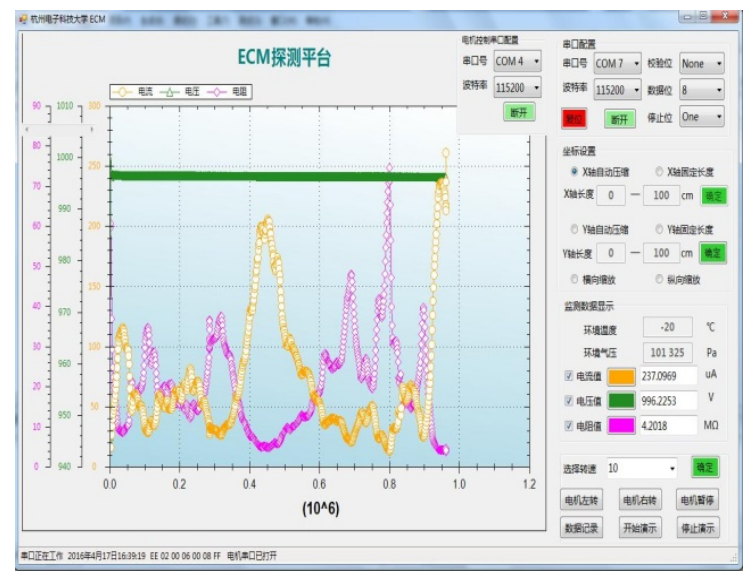

Fig.5. Human-computer interaction interface

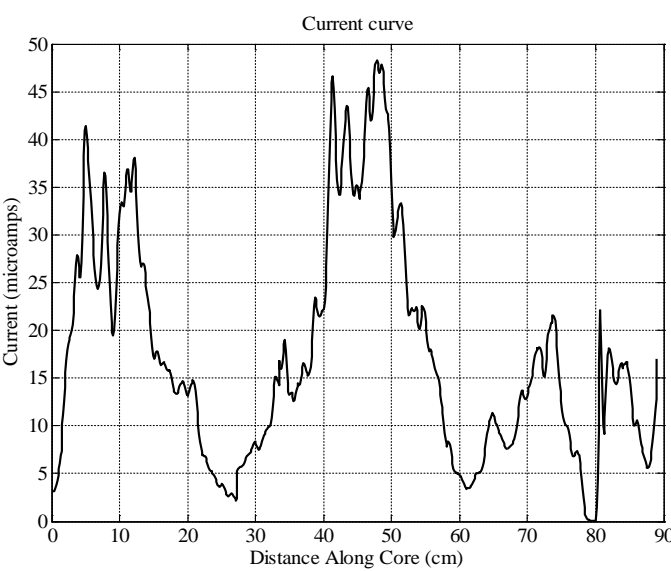

Fig.4. Current change

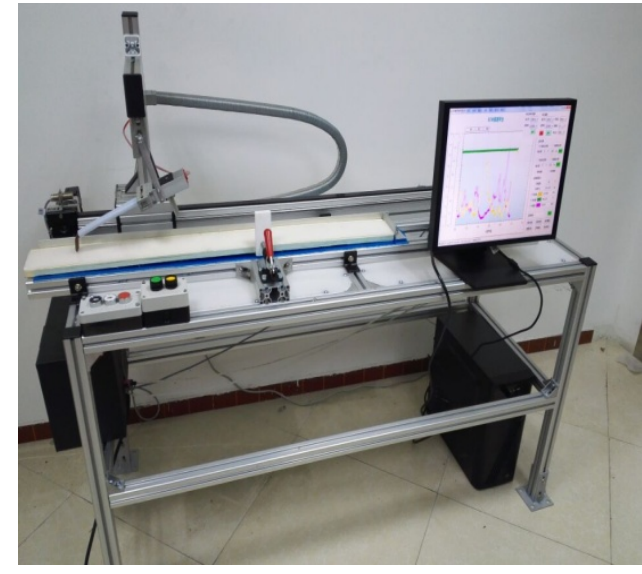

Fig.6. Overall constructed equipment

\section{Conclusion}

The experiment measured that the current can accurately reflect the conductivity of the ice core. The precision of the current can reach 0.1uA. And the current measurement ranges is from $0 \mathrm{uAto} 1000 \mathrm{uA}$. The resolution of displacement measured by grating scale can reach $0.005 \mathrm{~mm}$. In the environment of 40 degrees below zero, the system can work stably and reliably.

\section{Acknowledgement}

This work was partially supported by National High-tech R\&D Program of China (863 Progra m) (Grant No. 2011AA090401). We gratefully thank the anonymous reviewers whose insightful comments helped improve the presentation of this paper. 


\section{References}

[1] Souney J M, Twickler M S, Hargreaves GM, et al. Core handling and processing for the WAIS Divide ice-core project [J]. Annals of Glaciology, 2014, 55 (68) :15 - 26(12).

[2] Bo Sun, Tandong Yao, Jiancheng Kang, et al. Solid electrical conductivity measurement of polar ice cores and its environmental significance[J]. polar research, 1998(3):235-240.

[3] Hammer C U. Acidity of polar ice cores in relation to absolute dating, past volcanism, and radio-echoes[J]. Journal of Glaciology, 1980, 25(93):359-372.

[4] Hammer C U. Initial direct current in the buildup of space charges and the acidity of ice cores[J]. Journal of Physical Chemistry, 1983, 87(21):4099-4103.

[5] Yinke Dou, Xiaomin Chang, Jianmin Qin, et al. Application of Ice Thickness Monitoring Device Based on Resisitivity in Antarctic [J]. Journal of Taiyuan University of Technology, 2006, 37(4):454-456.

[6] Jun Dun. The practical application of STM32 encoder in the measurement of grating scale [J]. Science and technology innovation and Application, 2013(8):53-53.

[7] Yong Sang, Fengtao Li, Yuebang Dai, et al. The STM32 SCM Control System Design on Servo Motor Oriented [J]. Mechanical \& Electrical Engineering Technology, 2015(11):65-72. 